\title{
Editorial
}

\section{Hypertension- An update}

Hypertension is a global problem. Out of 7.1 billion people of the world; 1.2 billion is suffering from hypertension. Hypertension affects approximately $17.9 \%$ of the adult population in Bangladesh. The prevalence increases with age in both men and women.

Hypertension or high blood pressure is typically defined as $\mathrm{SBP} \geq 140 \mathrm{~mm} \mathrm{Hg}$ and/or DBP $\geq 90 \mathrm{~mm} \mathrm{Hg}$ on 2 or more occasions. In more than $95 \%$ of cases, a specific underlying cause of hypertension cannot be found. Such patients are said to have essential hypertension. Pathogenesis in such case is not clearly understood. In case of $5 \%$ cases there is specific cause, which is labeled as secondary hypertension.

World Hypertension Day is celebrated every year on 17th of May to raise the public awareness about the hypertension, its preventive measures and complications. The theme for 2015 was "Know Your Blood Pressure".

According to JNC'07 hypertension can be classified as-

\begin{tabular}{|c|c|c|}
\hline Classification & Systolic pressure $(\mathrm{mm}$ of $\mathrm{Hg})$ & Dia stolic pressure $(\mathrm{mm}$ of $\mathrm{Hg})$ \\
\hline Normal & $<120$ & $<80$ \\
\hline Prehypertension & $120-139$ & $80-89$ \\
\hline Hypertension- Stage 1 & $140-159$ & $90-99$ \\
\hline Hypertension- Stage 2 & $\geq 160$ & 100 \\
\hline
\end{tabular}

\section{Clinical evaluation of hypertension patient:}

Any person who comes with Hypertension or High Blood Pressure should be evaluated with following aims-

- How long a person is suffering from High Blood Pressure and also determine the level of BP.

- To exclude secondary causes of Hypertension.

- To determine the presence of target organ damage such as Heart, Brain, Kidney and eye etc.

- To search for other cardiac risk factors.

Minimum routine investigation for patient suffering from High Blood

\section{Pressure:}

G Family and personal history

G Full physical examination

Laboratory investigation

Urinalysis for Blood, Protein and Glucose.

a Blood Chemistry of Potassium, Creatinine, Blood glucose, Lipid profile

口 ECG

X-ray Chest PA View

$\square$ Echocardiography

- Further investigation should be guided from the history, examination and routine investigation.

\section{Management of Hypertension:}

Lifestyle modifications remain the cornerstone of hypertension management:

D Diet- low in salt, cholesterol and saturated fats and high in fresh fruits and vegetables.

Exercise- 30 to 60 minutes daily exercise, at least 5 days per week.

- Weight reduction in those who are overweight.

- A smoke free environment and tobacco cessation to reduce $\mathrm{CV}$ and cancer risks.

a Maintaining normal blood sugar levels.

- Encourage use of home blood pressure monitoring with approved device and proper technique.

\section{Drug Management:}

There are different classes of anti-hypertensive drugs including- Diuretics, Beta blockers, $\mathrm{Ca}$ channel blockers, ACE (-)/ARB, Alpha blockers, OthersMethyldopa, Hydralazine etc. Choice of drug/s should be based on-

- Socio-economic factors

- Cardiovascular risk factors

- Target organ damage, clinical cardiovascular disease, renal disease $\&$ diabetes

- Presence of other co-existing disorders that may either favor or limit the use of particular drug class

- Variation in individual response

- Possibility of interactions with other drugs

- Other compelling Indications 


\section{Latest JNC-8 recommendation for hypertension management:}

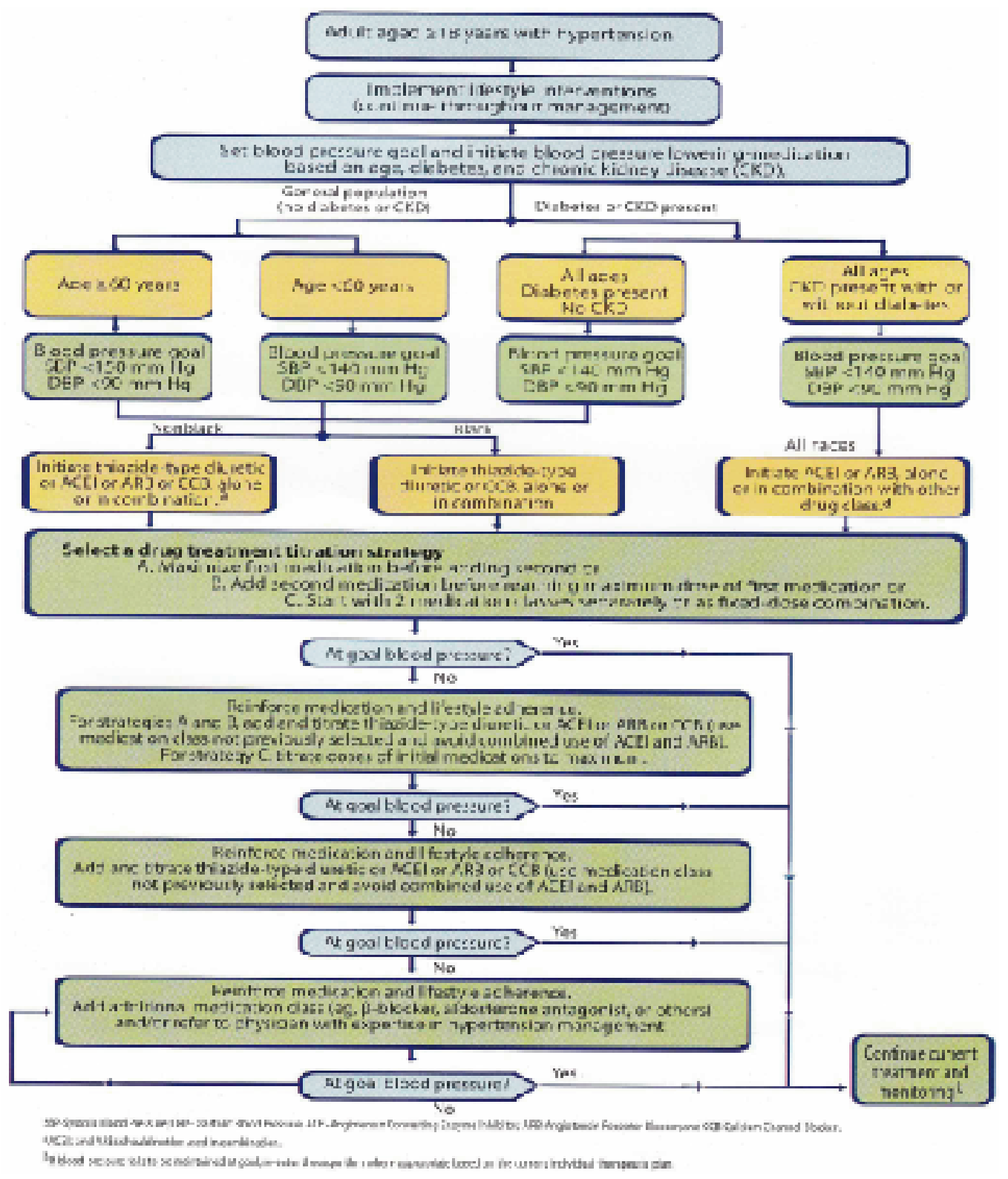

Resistant hypertension is defined as the failure to achieve goal blood pressure in patient who are on optimal doses of three or more anti-hypertensive drugs from different classes, ideally one of which is a diuretic. Newer treatment option- Renal denervation (RDN)

\section{Conclusion}

It is said that half among the hypertensive people are diagnosed; half of them gets treatment and half of them are well controlled. So both public and physician awareness is necessary. Before conclusion few points to be noted-

- Measure blood pressure whenever possible.

- Lowering blood pressure is the most powerful effect independent of drug choice.

- Some treatment is better than none

- Treatment needs to be continued.

- Remember to treat other risk factors.

- The aim is prevention of target organ damage.

\section{Prof. Dr. Md. Saiful Islam} MBBS, DTCD (D.U), MD, FACC (USA) Head of Cardiology, KYAMCH 